\title{
Retinal detachment due to macular holes
}

\author{
R. H. C. MARKHAM AND A. H. CHIGNELL \\ From the Ophthalmic Department, St Thomas's Hospital, London SEI
}

SUMmaRY Two unusual cases of retinal detachment are described in which macular holes were visible before a first operation but subsequently hidden owing to preretinal membrane and retinal fold formation at the macula. Only by very careful indentation at the macula at reoperation could these holes be identified and treated, with subsequent successful reattachment of the retina.

Macular holes are an uncommon cause of retinal detachment-1\% or less in most series. ${ }^{1}$ The difficulty of determining whether a hole at the macula is of full or partial thickness and, if the former, deciding whether the hole is the cause of the retinal detachment has been stressed by various authors. ${ }^{1-3}$ In view of these difficulties a definitive causal role in retinal detachment can be attributed to a macular role only if the detachment is confined to the posterior pole or, if more extensive, has been seen to have advanced from a posterior pole detachment. As surgical treatment aimed directly at the macular hole is hazardous and difficult, we use it as a primary procedure only if subretinal fluid does not extend to the periphery when first seen. If on initial examination subretinal fluid is found to extend to the ora serrata and a macular hole is present, a peripheral buckling procedure is performed, the assumption being that the detachment is caused by a peripheral hole whether detectable or not. If this fails to flatten the retina, a procedure directed at the macular hole is then performed.

The following 2 cases are reported to show that greater difficulty than usual may be encountered in cases in which preretinal fibrosis in the macular region is present to prevent an adequate view of a macular hole.

\section{Case reports}

C A S E 1

A 76-year-old man with low myopia ( -1.50 DS right and left), presented with a sudden decrease in right visual acuity to hand movements. A right temporal retinal detachment involving the macula was found extending to the ora serrata

Correspondence to Mr A. H. Chignell, St Thomas's Hospital, South Wing, Eye Department, Lambeth Palace Road, London SE1 7EH. with an equatorial horseshoe-shaped tear at 9 o'clock; there also appeared to be a fullthickness macular hole on slit-lamp examination. There was some immobility of retina due to formation of preretinal membrane resulting in a star fold temporal to the macula. The left eye was normal. The peripheral hole was treated with a radial $5 \mathrm{~mm}$ silicone sponge buckled over full-thickness sclera, cryotherapy, and drainage of subretinal fluid. There were no operative complications. Despite closure of the peripheral hole the detachment persisted with only minimal further reabsorption of subretinal fluid. Thickening of the preretinal membrane in the postoperative period caused the star-shaped fold to distort the macula and hide the original macular hole completely (Fig. 1). The detachment in-

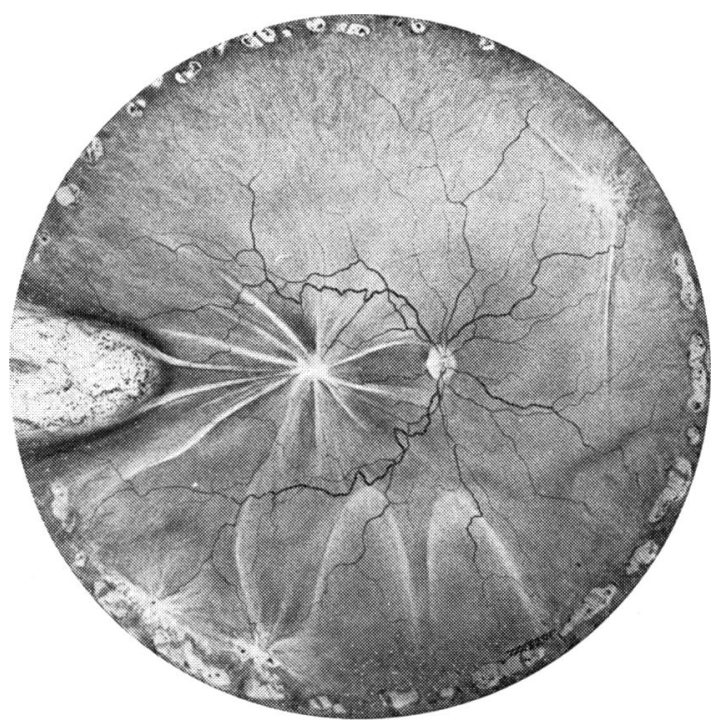

Fig. 1 Star-shaped fold obscuring the macular hole after the first operation. 
creased until it was almost total 1 month after operation. No further peripheral holes were found. Reoperation was advised, but only by careful indentation at the macula at the time of surgery could the original hole be detected. A $5 \mathrm{~mm}$ silicone sponge was placed over the macula and subretinal fluid was drained. No difficulty with exposure or suturing was encountered after detachment of the lateral rectus muscle, as there was no posterior staphyloma. There was progressive absorption of the remaining subretinal fluid; after 3 weeks the retina was flat and has remained so for the last 3 years.

\section{A S E 2}

A 56-year-old aphakic woman presented with a 3-day history of an increasing shadow in the upper temporal field of vision in the right eye. Six years previously she had a right extracapsular cataract extraction followed by capsulotomy for heterochromic cyclitis and secondary lens opacity. At the time of examination vision in the affected eye was reduced to hand movements. A few cells were found in the anterior chamber, and some old pigmented KP were present; the irides were typically heterochromic. A right temporal halfretinal detachment involving the macula was found extending to the ora serrata. It seemed that a full-thickness macular hole was present, but no peripheral hole was found. The left eye was normal.

It was impossible to exclude the possibility of a peripheral retinal hole, particularly as the view of the peripheral retina was partially obscured by capsular remnants, vitreous opacities, and poor pupillary dilatation. Therefore a peripheral buckling procedure was performed, and this took the form of an encircling silicone band. The peripheral ora was carefully searched with 360 degrees depression and cryotherapy in an unsuccessful attempt to identify holes at the time of operation. Subretinal fluid was not drained. In the postoperative few days a shallow annular choroidal detachment developed, but this subsided over the following 3 weeks. In spite of a satisfactory 1$2 \mathrm{~mm}$ high encircling ridge there was little reabsorption of subretinal fluid.

One month after operation the detachment was total.

Further search revealed no peripheral holes, and the original macular hole was no longer visible because of tight folds of retina due to preretinal membrane formation at the posterior pole. At reoperation the macular hole was found only after careful indentation. A $5 \mathrm{~mm}$ silicone sponge was placed over the macula; subretinal fluid was drained, and, as the immobile retina was still separated from the buckle, internal tamponage was achieved by $1 \mathrm{ml}$ of air injected into the vitreous through the pars plana. The retina was completely flat by the fifth postoperative day and has remained so for the past 3 years.

\section{Discussion}

Because of the presence of a peripheral hole in the first case and the presence of subretinal fluid extending to the periphery in the second, in both cases it was felt that the macular hole should not be treated directly as a first procedure. Nevertheless, that the macular hole had a causal role in the persistence of detachment is shown by the favourable effect of closure of the hole when buckled at a second operation.

After the first unsuccessful operation in both cases the previously noted macular holes could no longer be seen owing to the disposition of fixed retinal folds. Failure to seek and find a macular hole has been described as a cause, albeit rare, of failure of retinal detachment surgery. ${ }^{2}$ Had the macular holes not been seen at the time of the initial presentation, their presence may not have been suspected, and at reoperation a further unsuccessful operation could have been performed. When preoperative slit-lamp examination of the macula is unsatisfactory, owing either to opacities in the media or to retinal folds or fibrosis in the vicinity of the macula, it is suggested that careful indentation of the posterior pole is performed at the time of surgery, particularly if no peripheral holes have been found, as this may reveal a previously undetected macular hole.

We are grateful to Mr Terry Tarrant of the Audio-Visual Department of the Institute of Ophthalmology, London, for the illustration. Also we are grateful to Mrs Margaret Grice for her secretarial assistance.

This study was in part supported by the Iris Fund of St Thomas's Hospital.

\section{References}

1 Margerio RR, Schepens CL. Macular breaks 1. Diagnosis, etiology and observations. Am J Ophthalmol 1972; 74:219-32.

2 Scott JD. Macular holes and retinal detachment. Trans Ophthalmol Soc UK 1974; 94:319-24.

3 Leaver PR, Cleary PE. Macular hole and retinal detachment. Trans Ophthalmol Soc UK 1975; 95:145-7. 